\title{
Can Gasping be Used as a Tool to Determine Whether to Perform Compression-Only CPR versus Conventional CPR?
}

\author{
Eric M. Rottenberg, AAS
}

\author{
Correspondence: \\ Eric M. Rottenberg, AAS \\ 485 Harley Dr. Apt \# 414 \\ Columbus, Ohio 43202 USA \\ E-mail: rottenberg.1@osu.edu
}

\section{Conflicts of interest: none}

Keywords: Basic Cardiac Life Support; CPR; cardiopulmonary resuscitation; mouth-to-mouth resuscitation

\begin{abstract}
Abbreviations:
CCO: chest-compression only CPR: cardiopulmonary resuscitation EMS: Emergency Medical Services ICP: intracranial pressure OHCA: out-of-hospital cardiac arrest VF: ventricular fibrillation
\end{abstract}

Received: May 8, 2017

Accepted: July 23, 2017

Online publication: February 15, 2018

doi:10.1017/S1049023X18000031
Rottenberg EM. Can gasping be used as a tool to determine whether to perform compression-only CPR versus conventional CPR? Prehosp Disaster Med. 2018;33(2):225-226.

Witnessed arrest, favorable initial rhythm (ventricular fibrillation [VF]/ventricular tachycardia), and bystander cardiopulmonary resuscitation (CPR) are factors known to decrease mortality. However, recent studies have shown that in patients who suffer an out-of-hospital cardiac arrest (OHCA), chest-compression only (CCO) CPR can further increase survival among patients with a cardiac etiology, whereas conventional CPR can further increase survival among those with a non-cardiac etiology. ${ }^{1}$ However, according to Carter and Cone, ${ }^{1}$ Emergency Medical Services (EMS) personnel often lack objective and consistent criteria to assess whether an OHCA is of cardiac or non-cardiac etiology. They suggested that a more-specific consensus definition of cardiac etiology than that which currently exists in the Utstein template is needed to help better distinguish between cardiac and non-cardiac etiologies so that more optimal care can be delivered and mortality can be further decreased. However, the presence versus absence of gasping observed upon EMS arrival may be an important factor in the determination of cardiac versus non-cardiac etiologies and whether to perform CCO-CPR versus conventional CPR.

Studies in animals demonstrate that spontaneous gasping alone during VF cardiac arrest generates cardiac output, maintains more optimal amplitudes of $\mathrm{VF}$ waveforms, circulates blood to the brain, and decreases intracranial pressure (ICP). ${ }^{2-4}$ The lower the ICP is, the lower the resistance is to forward blood flow to the brain. Therefore, gasping can not only significantly improve the effectiveness of bystander CPR (and for that matter, EMSprovided $\mathrm{CPR}$ ), but also without any bystander $\mathrm{CPR}$, it can increase both survival and neurologic outcome. In fact, among patients who are found gasping, survival to hospital discharge is three times greater compared with those not found gasping. ${ }^{5,6}$ Among those who gasp and receive bystander CPR, survival to hospital discharge is more than four times greater. ${ }^{6}$ Results from a survey of survivors of OHCA in the Kanto region of Japan ${ }^{7}$ showed that 30-day favorable neurologic outcome for those who gasped (as evidenced by gasping observed upon arrival of EMS personnel) but received no CPR at all was virtually no worse than for those receiving CCO-CPR with gasping present upon EMS arrival (five percent versus six percent). Although it seems that there was not much of a difference (if any) in the 30-day favorable neurologic outcomes among those who were found gasping upon arrival of emergency rescuers but did not receive any $\mathrm{CPR}$ and those who were not found gasping (who were referred to as "apneic" patients) but did not receive any CPR (five percent versus two percent reported without any confidence intervals), there may be a significant difference. It is not known how many of these "apneic" patients were gasping but ceased at some point before arrival of emergency rescuers; however, the incidence of gasping observed during witnessed arrests before arrival of emergency rescuers has been previously shown to be $55 \%{ }^{5}$ In addition, when bystander CPR was performed with gasping present, patients receiving conventional CPR had far superior 30-day favorable neurologic outcome than those receiving $\mathrm{CCO}-\mathrm{CPR}$ (14\% versus six percent). A previous study also showed that gasping is associated with higher survival and good neurological outcome. ${ }^{8}$ One can argue that rescue breathing may have improved favorable neurological outcomes among those 
who gasped with a partially obstructed airway. Animal evidence suggests that arterial oxygenation and frequency of gasping during partially obstructed gasping with or without CCO-CPR would likely be critically reduced, less effective, and shorter lived. ${ }^{9,10}$ In anesthetized rats, even with the use of $100 \%$ supplemental oxygen, gasping through the natural airway while receiving $\mathrm{CPR}$ in the supine position significantly decreased the frequency of spontaneous gasping and arterial oxygenation compared with rats that had a specially designed oropharyngeal airway inserted. ${ }^{9}$ Therefore, arterial oxygenation and frequency of gasping during partially obstructed gasping with room air would likely be critically reduced. Moreover, as evidenced in intubated pigs allowed to breathe room air, even the incidence of unobstructed gasping is greatly reduced when the duration of $\mathrm{VF}$ without $\mathrm{CPR}$ is greater than three minutes; after four minutes, only $42 \%$ are still gasping and by the eighth minute (the average EMS response time in the United States), a mere eight percent. ${ }^{10}$ Partially obstructed gasping generates greater negative intrathoracic pressure than unobstructed gasping because of inspiratory impedance, which more greatly enhances perfusion of the heart and brain but significantly reduces oxygenation, thereby necessitating rescue breathing so that adequate oxygen is delivered to the heart and brain with or without chest compressions. As evidenced, gasping is an important confounding factor because it is associated with higher survival and good neurologic outcome.

References

1. Carter RM, Cone DC. When is a cardiac arrest non-cardiac? Prehosp Disaster Med. 2017;32(5):523-527.

2. Xie J, Weil MW, Sun S, Yu T, Tang W. Spontaneous gasping generates cardiac output during cardiac arrest. Crit Care Med. 2004;32(1):238-240.

3. Pernat AM, Weil MH, Bisera J, Tang WC, Fukui M. Spontaneous gasping maintains more optimal amplitudes of ventricular fibrillation waveforms during cardiac arrest. Circulation. 1998;17(Supplement):2511 [abstract].

4. Srinivasan V, Nadkarni VM, Yannopoulos D, et al. Spontaneous gasping decreases intracranial pressure and improves cerebral perfusion in a pig model of ventricular fibrillation. Resuscitation. 2006;69(2):329-334.

5. Clark JJ, Larsen MP, Culley LL, Graves JR, Eisenberg MS. Incidence of agonal respirations in sudden cardiac arrest. Ann Emerg Med. 1992;21(12):1464-1467.

6. Bobrow BJ, Zuercher M, Ewy GA, et al. Gasping during cardiac arrest in humans is frequent and associated with improved survival. Circulation. 2008;118(24):2550-2554.
In the above study of the incidence of agonal respirations during OHCA, ${ }^{5}$ it was found that gasping was observed in $46 \%$ of arrests caused by cardiac etiology compared with $32 \%$ in other etiologies $(\mathrm{P}<.01]$. Moreover, $56 \%$ percent of the arrests with $\mathrm{VF}$ had agonal activity compared with $34 \%$ of cases without VF $(\mathrm{P}<.001)$. Hence, the presence of gasping can, in part, be considered as an indicator of cardiac arrest of a cardiac etiology. However, because gasping is not universally observed in cardiac causes of OHCA and is observed in only 55\% of witnessed arrests, gasping alone cannot be used as a tool to better discriminate between cardiac and respiratory etiologies; age must also be considered.

Respiratory-related cardiac arrests typically occur in individuals of younger age compared with arrests due to cardiac causes, and hypoxia is most likely to occur before cardiac arrest, which can lead to lactic acidosis in the brain and subsequent brain swelling. ${ }^{11}$ The autopsy results of people who died as a result of OHCA in which brain swelling was a surrogate measure of respiratory-related cardiac arrest demonstrated a mean $(\mathrm{SD})$ age of $43(\mathrm{SD}=24)$ years versus $59(\mathrm{SD}=18)$ years for patients who did not develop brain swelling due to cardiogenic cardiac arrest $(\mathrm{P}<.01)$. Therefore, patients over the age of 59 who are found gasping are more likely to have had a cardiac arrest of cardiac origin and may benefit more from receiving CCO-CPR. All other patients, particularly those under the age of 43 found not to be gasping, should benefit more from receiving conventional $\mathrm{CPR}$.

7. SOS-KANTO Study Group. Cardiopulmonary resuscitation by bystanders with chest compression only (SOS-KANTO): an observational study. Lancet. 2007;369(9565): 920-926.

8. Martens P, Mullie A, Vanhaute O. Clinical status before and during cardiopulmonary resuscitation versus outcome in two consecutive databases. Belgian CPCR Study Group. Eur J Emerg Med. 1995;2(1):17-23.

9. Fukui M, Weil MH, Tang W, Yang L, Sun S. Airway protection during experimental CPR. Chest. 1995;108(6):1663-1667.

10. Menegazzi JJ, Check BD. Spontaneous agonal respiration in a swine model of out-ofhospital cardiac arrest. Acad Emerg Med. 1995;2(12):1053-1056.

11. Morimoto Y, Kemmotsu O, Kitami K, Matsubara I, Tedo I. Acute brain swelling after out-of-hospital cardiac arrest: pathogenesis and outcome. Crit Care Med. 1993; 21(1):104-110. 